\title{
Common Cognitive Control Processes Underlying Performance in Task-Switching and Dual-Task Contexts
}

\author{
Patricia Hirsch' ${ }^{1}$ Sophie Nolden ${ }^{1}$, Mathieu Declerck², and Iring Koch ${ }^{1}$ \\ ${ }^{1}$ Institute of Psychology, RWTH Aachen University, Aachen, Germany \\ ${ }^{2}$ Laboratoire de Psychologie Cognitive, Aix-Marseille Université \\ and Centre National de la Recherche Scientifique, Marseille, France
}

ABSTRACT

\section{KEYWORDS}

cognitive control,

task switching, dual tasks,

PRP effect

\begin{abstract}
In the present study, participants performed highly comparable task-switching and dual-task paradigms, and the paradigm-specific performance costs were analysed in the context of the commonly postulated core components of cognitive control (i.e., working memory updating, inhibition, and shifting). In the task-switching paradigm, we found switch costs (i.e., switch trials vs. repetition trials) and mixing costs (i.e., repetition trials in mixed-task blocks vs. single-task trials). In the dual-task paradigm, we observed a psychological refractory period (PRP) effect (i.e., Task 2 [T2] performance after short stimulus-onset asynchrony [SOA] vs. long SOA), dual-task costs (i.e., T2 dual-task performance with a long SOA in trials with a task repetition between Task 1 [T1] and T2 vs. single-task performance), and switch costs in T2 (i.e., dual-task performance in trials with a switch between $\mathrm{T} 1$ and $\mathrm{T} 2 \mathrm{vs}$. dual-task performance in trials with a repetition between T1 and T2). A within-subjects comparison of the performance costs showed a correlation between mixing costs and dual-task costs, possibly indicating shared underlying cognitive control processes in terms of working memory updating. Surprisingly, there was also a correlation between switch costs and the PRP effect, presumably suggesting that cognitive control, as opposed to passive queuing of response selection processes, contributes to the PRP effect.
\end{abstract}

\section{INTRODUCTION}

Humans behave in a goal-directed and adaptive way in continuously changing environments. Such behaviour is enabled by cognitive control, a construct also referred to as executive function (see Cohen, 2017, for a discussion about the interchangeable use of "cognitive control" and "executive function"), which is responsible for the regulation of cognitive processing in accordance with current task goals (e.g., Logan, 2003). Cognitive processes involved in the updating of current task goals, in their shielding against irrelevant information and action tendencies, and in the dynamic switching between goals or foci of attention when there is a change in internal needs or environmental circumstances have been identified as core components of cognitive control (i.e., working memory updating, inhibition, and shifting; Miyake et al., 2000).

From this short summary it is evident that cognitive control is required during multitasking (Miyake et al., 2000). Multitasking is defined as the activity of performing several tasks within a limited time period, and hence, characterized by the temporal overlap of cognitive processes involved in performing multiple tasks. Thus, it refers to task-switching situations, in which we switch rapidly between tasks, and dual-task situations, in which we perform tasks more or less simultaneously. Experimental studies showed that multiple-task processing-both in terms of task-switching and dual-tasking-results in performance costs relative to single-task processing (for reviews, see, e.g., Monsell, 2003; Pashler, 1994).

Corresponding author: Patricia Hirsch, Cognitive and Experimental Psychology, Institute of Psychology, RWTH Aachen University, Jägerstr. 17-19, D-52066 Aachen, Germany.

E-mail: patricia.hirsch@psych.rwth-aachen.de 
Performance costs occurring in multitasking contexts have been widely examined to get insights into cognitive control. As performance costs arising in task-switching and dual tasks have been primarily discussed and explained independently of each other, little behavioural data is currently available that would allow for conclusions about whether the same or different cognitive control components underlie performance costs in task-switching and dual-task contexts (see Koch, Poljac, Müller, \& Kiesel, 2018, for a recent integrative review).

In the present study, we addressed this open question of common cognitive control components by conducting a within-subject comparison across the performance costs arising in task-switching and dual-task contexts. To this end, we presented to participants highly comparable task-switching and dual-task paradigms.

\section{Cognitive Control}

Cognitive control enables complex cognition in nonroutine situations, such as in multitasking contexts. Miyake et al. (2000, Miyake \& Friedman, 2012; see also Lehto, Juujärvi, Kooistra, \& Pulkkinen, 2003) identified working memory updating, inhibition, and shifting as three core components of cognitive control, which they defined as follows.

Updating refers to processes of monitoring incoming information for task-relevance and manipulating working memory content according to task goals by replacing content that is no longer relevant with task-relevant content. Thus, this component describes processes beyond the passive information maintenance by assuming that working memory content is revised to successfully respond to changing environmental demands.

Inhibition involves deliberate controlled processes responsible for the suppression of irrelevant information and responses. It refers to the ability to suppress memory intrusions that are no longer task-relevant, prepotent and automatic responses, as well as task-irrelevant stimuli to the task at hand (Friedman \& Miyake, 2004).

Finally, shifting describes processes involved in the coordination of switching between various tasks, operations, and mental states (see, e.g., Kiesel et al., 2010, for a review). It is assumed to include the ability to engage and disengage appropriate task sets and to perform a new task in the context of proactive interference and negative priming (for proactive interference accounts, see, e.g., Allport, Styles, \& Hsieh, 1994). Inhibition of the previously activated tasks, operations, and mental states as well as updating of task-relevant information in working memory appears to be a substantial prerequisite for shifting (Best \& Miller, 2010; see, e.g., Koch, Gade, Schuch, \& Philipp, 2010, for a review). The shifting component refers to the coordination of cognitive processes, such as inhibition and updating, in order to ensure successful switching.

These core components of cognitive control are related but dissociable according to Miyake et al (2000). Against this background, the question arises as to which cognitive control components underlie performance in task-switching and dual-task contexts and whether performance in these contexts depends on shared components. The first step to answer this question is to examine the pattern of withinsubject correlations of performance costs across highly comparable task-switching and dual-task paradigms.

\section{Similarities and Differences Between Task-Switching and Dual- Tasking}

The reason for the traditionally independent investigation of taskswitching and dual-tasking may be that task-switching and dual-task paradigms usually differ decidedly with respect to how the tasks are performed. In task-switching paradigms, costs of strictly serial switching from one task to another are measured by asking subjects to perform two successive tasks, thereby following an instructed (alternating-runs paradigm; e.g., Rogers \& Monsell, 1995) or a random task sequence with a cue indicating the upcoming task to be performed (cueing paradigm; e.g., Meiran, 1996). In contrast, in dual-task paradigms, such as the psychological refractory period (PRP) paradigm (see, e.g., Pashler, 1994, for a review), subjects react with independent responses to two stimuli-Stimulus 1 (S1) and Stimulus 2 (S2) - which are presented with a varying stimulus onset asynchrony (SOA), and costs of performing two different tasks-Task 1 and Task 2 (T2)-in temporal overlap are assessed.

\section{TASK-SWITCHING}

In the task-switching paradigm, performance costs are measured in terms of mixing costs and switch costs (for a review, see, e.g., Kiesel et al., 2010). Mixing costs are calculated by comparing performance in repetition trials of mixed-task blocks against trials in single-task blocks (see, e.g., Koch, Prinz, \& Allport, 2005; Rubin \& Meiran, 2005). In mixed-task blocks, subjects repeat and switch tasks across trials, constituting an experimental setting where two task sets have to be held active, and incoming information has to be monitored for taskswitches and thus for task set updating. In contrast, single-task trials are performed in the context of constant task repetitions in order to obviate monitoring of incoming information to identify a task switch. Since none of the trial types contrasted in mixing costs have a switching requirement, the shifting component is held constant. Hence, mixing costs may mainly reflect working memory processes of task set maintaining and updating (Mayr, 2001).

Switch costs are calculated as the performance difference between switch trials and repetitions trials of mixed-task blocks (e.g., Allport et al., 1994; Rogers \& Monsell, 1995). In both trial types, incoming information has to be monitored with the objective of determining task-switches and updating task sets. Switch trials require the disengagement and the engagement of tasks, whereas in repetition trials the same task can be applied again. Thus, switch costs may primarily reflect the shifting component (Miyake et al., 2000).

Switching-related performance costs are often accounted for by assuming that a task set has to be activated to perform a task, and that the task set has to be reconfigured (e.g., deletion of the previous task set, implementation of a new task set), when the task changes (task set reconfiguration models; e.g., Meiran, 1996). Other researchers argue that to perform one of two tasks, it is necessary to inhibit the irrelevant task set. When the task switches, the persisting inhibition of the previously irrelevant task, but now intended task and the persisting activation of the previously relevant, but now unintended task have to 
be overcome (proactive interference models; e.g., Allport et al., 1994; see also Schuch \& Koch, 2003).

\section{DUAL-TASKING}

In dual-task paradigms, performance costs are assessed in terms of the PRP effect and dual-task costs. The PRP effect is calculated in dual-task blocks as T2 performance difference across short and long SOAs (Pashler, 1994). Since in dual-task studies T1 usually differs from T2 (see, e.g., Hirsch, Declerck, \& Koch, 2015; Lien, Schweickert, \& Proctor, 2003, for exceptions), the monitoring of information to update task sets in line with $\mathrm{T} 2$, as well as the disengagement and engagement of task sets in order to switch from T1 to T2, should be required regardless of the SOA. It thus remains unclear which specific component of cognitive control is isolated when comparing T2 performance across SOAs.

Dual-task costs are calculated by contrasting T2 performance in dual-task blocks with performance in single-task blocks. Since T2 performance in dual-task blocks includes costs evoked by the simultaneous selection of $\mathrm{T} 1$ and $\mathrm{T} 2$ responses, which are already reflected in the PRP effect, usually only the performance after long SOAs is considered for the measurement of dual-task costs (see Halvorson, Ebner, \& Hazeltine, 2013, for a discussion). Dual-task costs are assessed under conditions of varying working memory demands. In dual-task trials, two task sets have to be held active and incoming information has to be monitored to update task sets in accordance with T2, whereas in single-task trials, such monitoring processes are not necessary and only one task set has to be maintained. Thus, it is conceivable that, like mixing costs, dual-task costs might primarily depend on working memory updating and task set maintaining (see Miyake et al., 2000, for a similar hypothesis).

However, since in PRP studies, T1 usually differs from T2, the shifting component should also contribute to dual-task costs (Miyake et al., 2000). This is because in contrast to single-task trials, there is always the disengagement and engagement of task sets required when subjects switch from T1 to T2 in dual-task trials. Varying the task sequence within dual-task trials in such a way that there are switches and repetitions between $\mathrm{T} 1$ and T2 (e.g., T1-T2 switch: Task A as T1 and Task B as T2; T1-T2 repetition: Task B as T1 and as T2) and using only T1-T2 repetitions for the assessment of dual-task costs enables the disentanglement of the updating and shifting component.

A link between the specific cognitive control components proposed by Miyake et al. (2000) and dual-task-related performance costs has not often been drawn. Instead, dual-task-related performance costs are usually explained by processing capacity limitations and/or by functional limitations of the cognitive system.

The most popular model on capacity limitations is the response-selection bottleneck model (e.g., Pashler, 1994). According to this model, it is structurally impossible to select two responses in parallel, which is why T2 response selection has to wait until the availability of an all-ornone bottleneck at the stage of response selection (i.e., full capacity is allocated to $\mathrm{T} 1$ and then to $\mathrm{T} 2$ ), postponing $\mathrm{T} 2$ response selection (i.e., increasing the response time [RT] in T2 ) for the time during which the bottleneck is occupied by T1. Thus, this model accounts for dual-task related performance costs without referring to any cognitive control processes.

Of late, this model has gone through various modifications, all of which maintain the idea of a processing bottleneck at the stage of response-selection. For example, Hommel (1998; see also Lien \& Proctor, 2002; Schubert, Fischer, \& Stelzel, 2008) proposed to subdivide the response-selection stage into an automatic response-activation stage occurring immediately after stimulus identification and activating taskrelevant stimulus-response mappings, and a final response-selection stage which can be occupied by one task only. With sufficiently short SOAs, the response activation stages overlap for T1 and T2, enabling T2 characteristics to affect the duration of the response-activation stage of T1. This modification enables the response-selection bottleneck model to account for a variety of findings observed in dual-task research (e.g., response-response compatibility effects; e.g., Fischer \& Dreisbach, 2015; Hommel, 1998; Schuch \& Koch, 2004).

In contrast, according to capacity sharing models (e.g., Tombu \& Jolicoeur, 2003), response selection for two tasks may occur in parallel, but the processing capacity at the stage of response selection is limited. To optimize performance, response selection does not proceed in parallel for two tasks, but the capacity is allocated to the tasks in a graded manner, necessitating cognitive control processes for capacity scheduling.

Another line of models attributes dual-task related performance costs to functional limitations (e.g., computational constraints) of the cognitive system (see, e.g., Fischer \& Plessow, 2015, for a review). Such constraints occur when the same capacity is used "for different purposes by multiple processes" (Feng, Schwemmer, Gershman, \& Cohen, 2014 , p. 130). For instance, in the executive control of the theory of visual attention (ECTVA) model (Logan \& Gordan, 2001; see also the executive-process interactive control [EPIC], model; Meyer \& Kieras, 1997), the PRP effect is due to a strategic decision for serial processing in order to resolve the dual-task-binding problem. This problem refers to the difficulty of distinguishing which response goes with which task when running the evidence accumulation process for two tasks in parallel. Thus, according to this model, T1 and T2 are performed serially, to minimize the risk of unwanted response reversal (see also Schubert, 2008 , for a discussion on the role of order control in dual-tasking).

\section{TEMPORAL ASPECTS OF MULTITASKING}

Time intervals have a crucial impact on performance costs in both paradigms. The manipulation of the SOA is the key element of the PRP paradigm. The SOA defines the degree of temporal overlap in T1 and T2 processing. Task-switching experiments also offer the option of varying time components, including, among others, the responsestimulus interval (RSI). The RSI determines the time period between the processing of tasks ${ }^{1}$.

\section{RELATING TASK-SWITCHING AND DUAL-TASKING}

At first sight, task-switching and dual-task paradigms appear to differ greatly from each other. However, in both paradigms, perform- 
ance costs are affected by the duration of specific time intervals, and they are explained by assuming that there are cognitive processes prohibiting a strong parallel activation of two task sets on the one hand and a fully parallel response selection on the other (Koch et al., 2018). However, switching-related performance costs and dual-task-related performance costs differ in the sense that they arise in task-switching paradigms under situations of strictly serial-task processing, whereas in dual-task paradigms they occur in situations of temporally overlapping task processing.

Despite the commonalities between task-switching and dual-tasking, there are only a few studies and theoretical considerations about how the performance costs in task-switching and dual-tasking might be related (e.g., Band, Jolicoeur, Akyürek, \& Memelink, 2006; Oriet \& Jolicoeur, 2003; Pashler, 2000; Sigman \& Dehaene, 2006). For instance, by implementing T1-T2 switches and T1-T2 repetitions into dual-task trials, Band and van Nes (2006) as well as Lien et al. (2003) observed, in addition to a PRP effect, switch costs in T2. The cost was comparable across SOAs. Lien et al. (2003) concluded from the switch costs in T2 that time is needed for processes involved in the disengagement of $\mathrm{T} 1$ and the engagement of T2, and thus, that the shifting component is involved in dual-tasking (see also Liepelt, Strobach, Frensch, \& Schubert, 2011, for similar conclusions derived from studies on practice effects in dual-tasking; for a review, see, e.g., Strobach \& Schubert, 2017). They argued that the missing absorption of switch costs in T2 during the waiting period for the availability of the response-selection bottleneck (i.e., additivity of SOA and T1-T2 transition) suggests that these processes, at least partially, occur after response selection for $\mathrm{T} 1$ and before response selection for $\mathrm{T} 2$, and modified the response-selection bottleneck accordingly.

Using a latent variable analysis, Miyake et al. (2000) confirmed that the shifting component contributes to switch costs. However, they found dual-task costs to be not related to any of the core components and concluded cautiously, based on these null findings, that dual-task costs rely on an independent component of cognitive control which is not related to the proposed core components. Mixing costs, the PRP effect, and their underlying cognitive control components were not examined in their study.

In the present study, we assessed, besides switch costs and dualtask costs, mixing costs and the PRP effect to analyse these costs in the context of the core components of cognitive control. The goal was to identify shared and paradigm-specific cognitive control components by measuring switching-related and dual-task-related performance costs in highly comparable paradigms and analyzing cross-paradigm correlations between them.

\section{THE PRESENT STUDY}

To examine whether performance costs in task-switching and dualtask paradigms have a common source, we designed a task-switching and dual-task paradigm as similarly as possible using the same stimuli, tasks, and responses. In the task-switching paradigm, subjects performed single-task and mixed-task conditions with a short and long
RSI, whereas in the dual-task paradigm, subjects performed single-task and dual-task conditions with a short and long SOA. The dual-task conditions included both T1-T2 switches and T1-T2 repetitions. With these highly comparable paradigms, we measured the paradigm-specific performance costs and, in order to gain first insights into paradigmspecific and common cognitive control components, we subjected the costs to a correlation analysis (see, e.g., Declerck, Grainger, Koch, \& Philipp, 2017, for the use of correlation analyses in the study of shared cognitive control processes involved in task-switching and language switching).

In the task-switching paradigm, we expected to find mixing costs and switch costs. In the PRP paradigm, we expected a PRP effect, T2 switch costs, and dual-task costs. Regarding dual-task costs, we argue that $\mathrm{T} 2$ performance in $\mathrm{T} 1-\mathrm{T} 2$ repetition trials represents a more appropriate condition to be compared with single-task conditions. Our rationale is that in T1-T2 repetitions, T2 performance is unaffected by task switches, like the performance in single-task trials. Therefore, we calculated dual-task costs by contrasting T2 performance in T1-T2 repetition trials with single-task performance, considering only trials with a long SOA. Moreover, we hypothesized a positive correlation between mixing costs and dual-task costs, possibly reflecting shared underlying cognitive control processes in terms of updating. In the literature, a specific cognitive control component contributing to the PRP effect has not yet been identified. Therefore, hypotheses about the PRP effect and its relation to other performance costs cannot be clearly derived at the current point.

\section{Method}

\section{PARTICIPANTS}

Thirty-two participants (29 women, age range: $17-38$ years; $M_{\text {age }}$ $=22.7$ years) took part in the experiment. One additional participant was tested, but because of an excessive error rate of $>45 \%$, the data was replaced by a new data set, leaving 32 data sets for the analyses. All participants had normal or corrected-to-normal vision.

\section{STIMULI, TASKS, AND RESPONSES}

The stimulus material consisted of a fixation cross (+), an asterisk, digits from 1 to 9 (except 5), and capital letters, including the consonants $G, K, M$, and $R$ as well as the vowels $A, E, I$, and $U$. The stimuli were presented in white $20 \mathrm{pt}$ Arial font on a black screen placed at a distance of approximately $50 \mathrm{~cm}$. The fixation cross was presented at the centre of the screen, where it stayed throughout the entire experiment. Digits and letters were displayed $3 \mathrm{~cm}$ to the left and to the right of the fixation cross.

Subjects were asked to categorize digits as odd or even and letters as consonants or vowels. They used their index or the middle finger of the hand corresponding to the stimulus presentation location for response execution. Responses to stimuli appearing to the left of the fixation cross were made with the $Y$ and $X$ keys of a QWERTY keyboard. The $N$ and $M$ keys were used as response keys for stimuli presented to the right of the fixation cross. The stimulus-response mapping for both tasks was counterbalanced across participants. 


\section{PROCEDURE}

The experiment was run in a single session, with one participant at a time, and took about $60 \mathrm{~min}$. Instructions were given both in verbal and in written form, and emphasized speed and accuracy. The experiment consisted of a task-switching and a dual-task part with counterbalanced order across participants. Both the task-switching and the dual-task part comprised the following experimental blocks in the stated order-one single-task block of 41 trials for each task type, four mixed-task blocks in the task-switching part or dual-task blocks in the dual-task part, of 81 trials each, and one single-task block of 41 trials for each task type. The first single-task blocks were preceded by practice blocks of six trials, and the first mixed-task or dual-task blocks were preceded by a practice block of 12 trials. Importantly, the task type for the first single-task block was counterbalanced across subjects.

In the task-switching part, the stimuli appeared alternately to the left and right of the fixation cross, starting at the left side. They remained on the screen until a response was executed. The next stimulus was displayed after a random RSI of $100 \mathrm{~ms}$ or $600 \mathrm{~ms}$.

In single-task blocks of the dual-task part, an asterisk, which served as task-irrelevant S1, appeared to the left of the fixation cross, followed by a task-relevant $\mathrm{S} 2$ to the right of the fixation cross after a random SOA of $100 \mathrm{~ms}$ or $600 \mathrm{~ms}$. In dual-task blocks, the asterisk was replaced by a task-relevant $\mathrm{S} 1$. Both stimuli disappeared after T2 response execution. The next trial began $1 \mathrm{~s}$ later (intertrial interval, ITI).

The stimulus presentation was random with the restriction that all stimuli were displayed equally often (i.e., when including also practice trials) and that there were no immediate stimulus repetitions ${ }^{2}$. In the dual-task part, S1 and S2 varied independently of each other. However, we controlled that the number of repetitions and switches between T2 of the previous trials and $\mathrm{T} 1$ in the current trial was the same.

\section{DESIGN}

For the task-switching part, we specified two non-orthogonal $2 \times 2$ contrasts, including a mixing-cost contrast and a switch-cost contrast. The independent within-subject variables were RSI (100 ms vs. 600 $\mathrm{ms}$ ) and trial type. In the mixing-cost contrast, we contrasted performance in repetition trials of mixed-task blocks with that in single-task trials, whereas in the switch-cost contrast, we compared performance in switch trials with that in repetition trials of mixed-task blocks.

For the dual-task part, we analysed the effects of T1-T2 sequence and SOA separately for $\mathrm{T} 1$ and $\mathrm{T} 2$ based on a $2 \times 2$ repeated-measures design with the independent within-subject variables of T1-T2 sequence (T1-T2 switch vs. T1-T2 repetition) and SOA (100 ms vs. 600 $\mathrm{ms})$. Finally, to measure dual-task costs, we contrasted performance across single-task trials and dual-task trials with long SOA which included a task repetition between $\mathrm{T} 1$ and $\mathrm{T} 2$.

To deal with speed-accuracy trade-offs with respect to the main effects of RSI and SOA and with respect to the interaction of RSI and trial type, we do not report separate analyses for RT and error rates but linear integrated speed-accuracy scores (LISAS; Vandierendonck, 2017) as the dependent variable. The LISAS integrate speed and accuracy, resulting in RT scores that are corrected for the number of errors committed (for the mean RT data and the error data, see Table Al in the Appendix; Bruyer \& Brysbaert, 2011; Vandierendonck, 2017).

\section{RESULTS AND DISCUSSION}

Practice blocks, the first trial in each block, and trials following an error were eliminated from all data analyses. Trials with an erroneous response and trials deviating more than 3 SDs from each individual's mean RT per condition (task-switching part: $1.9 \%$ of single-task trials, $2.0 \%$ of mixed-task trials; dual-task part: $1.9 \%$ of single-task trials, $1.9 \%$ of $\mathrm{T} 1$ in dual-task trials, and $2.0 \%$ of $\mathrm{T} 2$ in dual-task trials) were additionally discarded from the RT analysis. We report the results separately for the task-switching and the dual-task part (see Figure 1 and Figure 2), followed by a joint analysis for correlations.

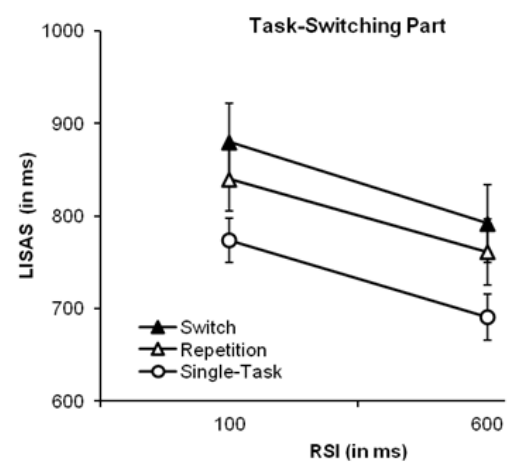

|FIGURE 1.

Linear Integrated Speed-accuracy Scores (LISAS; in ms) in the task-switching part as a function of trial type (switch trials, repetition trials, and single-task trials) and response stimulus interval (RSl; 100 ms vs. 600 ms). Error bars represent the standard error.

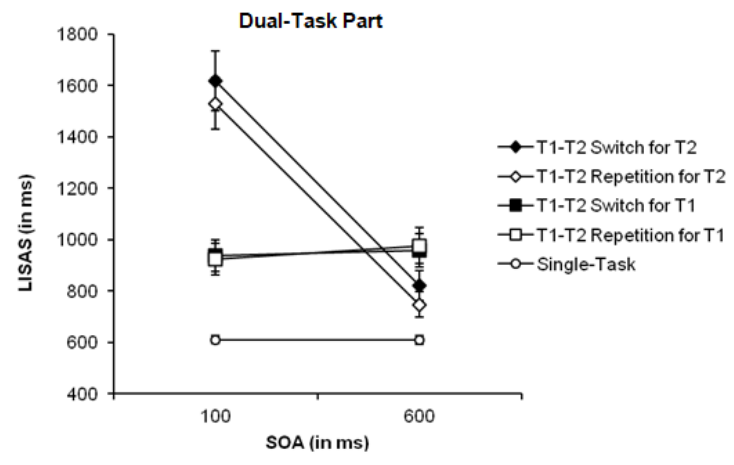

\section{| FIGURE 2.}

Linear Integrated Speed-accuracy Scores (LISAS; in ms) in the dual-task part as a function of trial type (T1-T2 switch trials, T1-T2 repetition trials, and single-task trials) and stimulus onset asynchrony (SOA; 100 ms vs. 600 ms). Error bars represent the standard error. 


\section{Task-Switching Performance}

Mixing costs. There were significant main effects of trial type, $F(1,31)$ $=10.31, p<.01, \eta_{\mathrm{p}}{ }^{2}=.25$, and RSI, $F(1,31)=61.87, p<.001, \eta_{\mathrm{p}}{ }^{2}=.67$. Response times were higher in repetition trials of mixed-task blocks than in single-task trials (800 ms vs. $733 \mathrm{~ms}$ ) and with short RSI than with long RSI ( $807 \mathrm{~ms}$ vs. $726 \mathrm{~ms}$ ), reflecting mixing costs of $67 \mathrm{~ms}$ and an RSI effect of $81 \mathrm{~ms}$. The interaction of trial type and RSI was nonsignificant, $F<1$.

Switch costs. There was a significant main effect of trial type, $F(1$, $31)=11.06, p<.01, \eta_{\mathrm{p}}{ }^{2}=.26$, indicating slower responses in switch trials than in repetition trials ( $836 \mathrm{~ms}$ vs. $800 \mathrm{~ms}$; switch costs: $36 \mathrm{~ms}$ ). The main effect of RSI was significant, too, $F(1,31)=47.19, p<.001, \eta_{\mathrm{p}}{ }^{2}$ $=.60$. Responses were slower with short RSI than with long RSI (860 ms vs. $776 \mathrm{~ms}$; RSI effect: $84 \mathrm{~ms}$ ). The interaction of trial type and RSI was non-significant, $F<1$.

\section{Dual-Task Performance}

Task 1. For T1, there was no main effect of T1-T2 sequence, $F<1$. However, the main effect of SOA was significant, $F(1,31)=7.11, p<$ $.05, \eta_{\mathrm{p}}{ }^{2}=.19$, reflecting faster responses with short SOA than with long SOA (932 ms vs. $968 \mathrm{~ms}$ ). Moreover, there was a nonsignificant trend towards an interaction of T1-T2 sequence and SOA, $F(1,31)=2.99, p$ $=.09, \eta_{\mathrm{p}}{ }^{2}=.09$, suggesting faster responses with short than with long SOA in repetition trials (924 ms vs. $977 \mathrm{~ms} ; t[31]=-2.93, p<.01, d=$ $0.14)$, but no RT differences across SOAs in switch trials $(939 \mathrm{~ms}$ vs. $959 \mathrm{~ms} ; t[31]=-1.38, p=.18, d=0.06$ ).

Task 2. For T2, there was a significant main effect of T1-T2 sequence, $F(1,31)=16.60, p<.001, \eta_{\mathrm{p}}{ }^{2}=.35$, reflecting slower responses in T1-T2 switch trials than in T1-T2 repetition trials $(1,220 \mathrm{~ms}$ vs. $1,139 \mathrm{~ms}$ ), and thus, switch costs in T2 of $81 \mathrm{~ms}$. Moreover, the main effect of SOA was significant, $F(1,31)=141.46, p<.001, \eta_{\mathrm{p}}{ }^{2}=.82$. Responses were slower with short SOA than with long SOA (1,575 ms vs. $784 \mathrm{~ms}$; PRP effect: $791 \mathrm{~ms})$. The interaction of T1-T2 sequence and SOA was nonsignificant, $F<1$.

Dual-task costs. A one-tailed $t$ test showed that in trials with a long SOA, responses were slower in T1-T2 repetition trials than in singletask trials (747 ms vs. $610 \mathrm{~ms}$ ), $t(31)=3.18, p<.01, d=0.65$, resulting in dual-task costs of $137 \mathrm{~ms}$.

\section{Interim Summary}

To summarize, in the task-switching part, we observed mixing costs and switch costs. Performance was worse with short than with long RSI. However, there was no effect of RSI on mixing costs and switch costs. In the dual-task part, we observed an SOA effect in $\mathrm{Tl}^{3}$, a PRP effect, dual-task costs, and switch costs in T2. Switch costs in T2 were about the same across SOAs, possibly indicating that task set reconfiguration or/and the modification of task set activation levels take place after response selection for $\mathrm{T} 1$ (for an accordingly modified responseselection bottleneck model, see Lien et al., 2003).

\section{Comparison of Paradigm-Specific Costs}

We obtained performance costs in both paradigms. To examine whether there is a relationship between these paradigm-specific performance costs, suggesting overlapping cognitive control processes, we calculated mixing costs, switch costs, dual-task costs (i.e., only after a long SOA), switch costs in T2, and the PRP effect (i.e., averaged over T1-T2 switches and repetitions) individually for each participant and subjected them to a correlation analysis (see Table 1).

Across paradigms, there was a significant relationship between mixing costs and dual-task costs, $r(32)=.43, p<.05$ (see Figure 3 ), indicating that the magnitude of mixing costs increased with increasing dual-task costs. Furthermore, mixing costs correlated positively with the PRP effect, $r(32)=.50, p<.01$, and with switch costs in T2, $r(32)=$ $.67, p<.001$. The correlation between switch costs and the PRP effect

\section{TABLE 1.}

Correlation Coefficients Between Performance Costs Assessed in the Task-Switching (TS) Part and Performance Costs Measured in the Dual-task (DT) Part for Linear Integrated Speed-Accuracy Scores (LISAS)

\begin{tabular}{cccccc}
\hline & $\begin{array}{c}\text { Mixing } \\
\text { costs in } \\
\text { TS }\end{array}$ & $\begin{array}{c}\text { Switch } \\
\text { costs in } \\
\text { TS }\end{array}$ & $\begin{array}{c}\text { Dual- } \\
\text { task } \\
\text { costs in } \\
\text { DT }\end{array}$ & $\begin{array}{c}\text { T2 } \\
\text { switch } \\
\text { costs in } \\
\text { DT }\end{array}$ & $\begin{array}{c}\text { PRP } \\
\text { effect in } \\
\text { DT }\end{array}$ \\
\hline $\begin{array}{c}\text { Mixing } \\
\text { costs in TS } \\
\text { Switch }\end{array}$ & 1 & $.48^{* *}$ & $.43^{*}$ & $.67^{* * *}$ & $.49^{* *}$ \\
$\begin{array}{c}\text { costs in TS } \\
\text { Dual-task }\end{array}$ & 1 & .27 & $.64^{* * *}$ & $.63^{* * *}$ \\
costs in DT & & & 1 & $.42^{*}$ & .35 \\
T2 switch & & & & & \\
costs in DT & & & & 1 & $.60^{* * *}$ \\
PRP effect & & & & & 1 \\
in DT & & & & & \\
\hline
\end{tabular}

Note. ${ }^{*} p<.05,{ }^{* *} p<.01,{ }^{* *} p<.001 . \mathrm{PRP}=$ Psychological Refractory Period

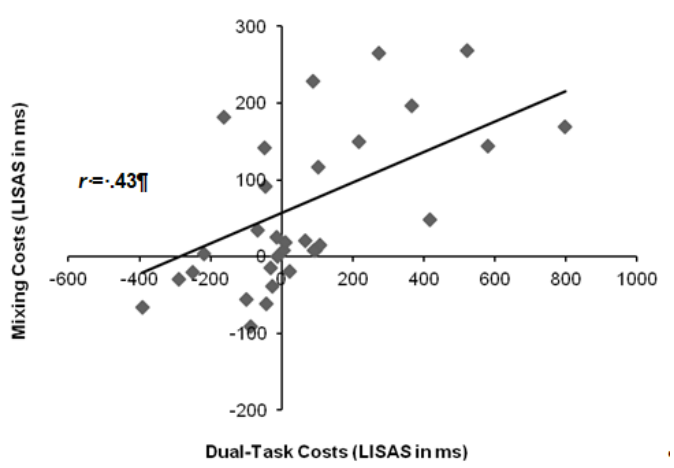

FIGURE 3.

Correlation between mixing costs (i.e., averaged over short and long response-stimulus intervals, RSIs) and dual-task costs (i.e., only for long stimulus-onset asynchrony, SOA). LISAS = Linear Integrated Speed-Accuracy Scores. 


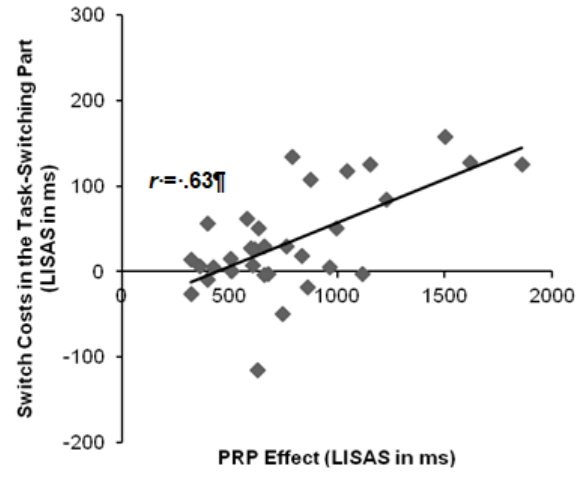

FIGURE 4.

Correlation between switch costs and the psychological refractory period (PRP) effect. LISAS = Linear Integrated Speed-Accuracy Scores.

(i.e., averaged over T1-T2 switch and T1-T2 repetition trials) was also significant, $r(32)=.63, p<.001$. Switch costs increased when the PRP effect increased (see Figure 4). Surprisingly, this correlation remained significant, even when using only T1-T2 repetitions for the calculation of the PRP effect, $r=.59, p<.001$. Moreover, switch costs were related to switch costs in T2, $r(32)=.64, p<.001$.

Regarding the relationships between performance costs measured within the same paradigm, we found positive correlations between mixing costs and switch costs, $r(32)=.48, p<.01$, and between the PRP effect and switch costs in T2, $r(32)=.60, p<.001$. Moreover, there was a trend towards a relationship between dual-task costs and the PRP effect, $r(32)=.35, p=.051$.

\section{DISCUSSION}

In the present study, we evaluated correlations between the performance costs arising in task-switching and dual-task paradigms, to examine whether shared cognitive control components underlie performance impairments in these multitasking contexts. In the task-switching part, we found mixing costs and switch costs. In the dual-task part, we observed dual-task costs, a PRP effect, and switch costs in T2. In line with our hypothesis, there was a positive correlation between mixing costs and dual-task costs. Moreover, there were correlations between switch costs, switch costs in T2, and the PRP effect.

\section{Underlying Mechanisms of Task-Switching and Dual-Task Performance}

Cognitive control enables successful performance in task-switching and dual-task contexts (Meyer \& Kieras, 1997). The current study did not allow specifying these cognitive control processes directly; however, it was possible to explore whether the observed performance costs rely on related or unrelated components of cognitive control.

\section{TASK SET SHIFTING}

The correlation analysis showed that switch costs in the taskswitching part correlated with switch costs in T2 and the PRP effect. Like switch trials in the task-switching part, T1-T2 switch trials in the dual-task part possibly also required a task set shift, more specifically between the task set of $\mathrm{T} 1$ and that of $\mathrm{T} 2$. This implies that by contrasting performance across T1-T2 switches and repetitions, and thus by calculating switch costs in T2, the shifting component might be isolated. Given that switch costs in the task-switching part and the dualtask part are indices of the shifting component of cognitive control, the correlations between switch costs, switch costs in T2, and the PRP effect might indicate that the shifting component contributes to the PRP effect.

Importantly, the effects of SOA and task transition in T2 were additive, reflecting comparable switch costs in T2 across SOAs. For response-selection bottleneck models, this finding means that shifting occurs after response selection for $\mathrm{T} 1$ and before response selection for $\mathrm{T} 2$, requiring shifting processes to wait until the response for $\mathrm{T} 1$ is selected with both short and long SOAs, thus impairing T2 performance across SOAs to the same degree. Considering such processing streams for $\mathrm{T} 1$ and $\mathrm{T} 2$, the effect of shifting should be eliminated and performance differences across SOAs should only be attributable to passive response-selection queuing after subtracting $\mathrm{T} 2$ performance with long SOA from that with short SOA. Hence, a response-selection stage without any shifting processing and a following stage responsible for the complete task set shift procedure cannot account for the correlation of the PRP effect with markers of the shifting component.

This conclusion is further reinforced by the observation that the correlation of switch costs and the PRP effect remained significant when using only T1-T2 repetition trials for the calculation of the PRP effect. This correlation suggests that, even when a task is repeated across $\mathrm{T} 1$ and $\mathrm{T} 2$, two separate task sets have to be activated (i.e., the same task set twice; see, e.g., Logan \& Gordon, 2001), and that switching between different components of these task sets (e.g., response mappings) is necessary to perform T1 and T2 in a temporal overlap (e.g., Duncan, 1995). This finding clearly contradicts the assumptions by Pashler (2000) who argued that the passive queuing of the response selection for T2 is the single source of the PRP effect and that the occurrence of the PRP effect in trials where a task is repeated across T1 and T2 speaks against the involvement of shifting processes.

\section{RESPONSE INHIBITION}

PRP trials with a short SOA, where two stimuli are presented almost simultaneously, resemble bivalent stimuli in task-switching, thereby also affording the application of the competing task. Thus, when there is a response-set overlap, such as the conceptual overlap in the present study, they also activate the competing response, thereby exogenously cueing between-task competition and crosstalk (e.g., Kiesel et al., 2010; Koch et al., 2018).

In PRP studies, response-response (R-R) compatibility effects are usually interpreted as empirical evidence for competition between T1 and T2 (e.g., Fischer \& Dreisbach, 2015; Hommel, 1998; Schuch \& 
Koch, 2004). They are measured as the performance decrement in R-R incompatible trials (i.e., response switch; e.g., left key in $\mathrm{T} 1$ and right key in T2) compared to R-R compatible trials (e.g., response repetition; e.g., right key in $\mathrm{T} 1$ and $\mathrm{T} 2$ ).

To explore if competition between $\mathrm{T} 1$ and $\mathrm{T} 2$ contributed to the PRP effect, we ran an additional analysis with the independent variables of T1-T2 sequence, SOA, and R-R compatibility. Whereas there was no effect of R-R compatibility on $\mathrm{T} 1$ performance, $p>.38$, in each instance, the interaction of T1-T2 sequence and R-R compatibility was significant for T2, $F(1,27)=17.18, p<.001, \eta_{p}{ }^{2}=.40(p>.23$, for all other cases). In repetition trials, responses were slower with $\mathrm{R}-\mathrm{R}$ incompatible than with R-R compatible trials (1,187 ms vs. $1,109 \mathrm{~ms}$; R-R compatibility effect: $78 \mathrm{~ms}$, post hoc one-tailed $t$ test: $t$ [27] $=2.43$, $p<.05, d=0.17)$. In switch trials, however, the opposite data pattern was observed (1,278 ms vs. 1,208 ms; reversed compatibility effect: 70 ms, post hoc one-tailed $t$ test: $t[27]=-3.07, p<.01, d=0.14$ ).

In numerous task-switching studies, R-R compatibility, in this domain referred to as response repetition, has also been observed to be beneficial when the task is repeated and detrimental when the task is switched (e.g., Koch, Frings, \& Schuch, 2017; Koch, Schuch, Vu, \& Proctor, 2011; Schuch \& Koch, 2010). Several models have been proposed to account for this interaction (cf. Druey, 2014; Rogers \& Monsell, 1995).

One group of models that is linked to the inhibition component of cognitive control (e.g., Druey \& Hübner, 2008) is especially important in the context of the present study. These models postulate that to prevent perseveration tendencies, responses are inhibited immediately after being selected. According to these models, there are no responseresponse repetition costs in task repetition trials because response repetitions within task repetition trials are accompanied by a stimuluscategory repetition that primes the inhibited response, which, in turn, weakens the inhibition, leading to improved performance relative switch trials.

These mechanisms of response inhibition and stimulus-category priming can be transferred from serial task processing to temporally overlapping task processing. The opposite effects of R-R compatibility across T1-T2 switches and repetitions, along with its independency from SOA, suggest that $\mathrm{T} 1$ responses are inhibited after being selected. Thus, response inhibition might be a part of either the response-selection stage or of an additional stage that follows $\mathrm{T} 1$ response selection and postpones $\mathrm{T} 2$ response selection until the response for $\mathrm{T} 1$ is inhibited (see also the ECTVA model by Logan \& Gordon, 2001).

Importantly, there was also an interaction of task sequence and response sequence (i.e., R-R compatibility) in the task-switching part, $F(1,31)=4.89, p<.05, \eta_{\mathrm{p}}{ }^{2}=.14$. Response repetition led to a cost, moreso in task-switch trials ( $878 \mathrm{~ms}$ vs. $835 \mathrm{~ms}$ ) than in task-repetition trials ( $822 \mathrm{~ms}$ vs. $811 \mathrm{~ms}$ ). Post hoc one-tailed $t$ tests showed that response repetition costs were significant for task-switch trials, $t(31)$ $=3.43, p<.01, d=0.18$, but nonsignificant for task-repetition trials, $t(31)=0.83, p=.21, d=0.05$. The lacking response repetition effect in task repetitions might be accounted for by the use of univalent stimuli, which have been shown to exhibit weaker effects of response sequence than bivalent stimuli (e.g., Grzyb \& Hübner, 2013; Hübner \& Druey, 2006). In this light, it is conceivable that the correlation between the PRP effect and switch costs reflects stimulus-category priming in combination with response inhibition.

Response inhibition and stimulus-category priming, however, cannot account for the correlation of dual-task costs and mixing costs. As indicated by R-R compatibility effects ( $106 \mathrm{~ms}$; two-tailed $t$ test: $t$ [27] = $3.06, p<.01, d=0.35)$, there was between-task competition in T1-T2 repetition trials with long SOA. In contrast, in single-task trials, only one stimulus was presented, eliminating the potential effects of bivalent stimuli. Thus, dual-task costs might be inflated by mechanisms involved in the resolution of between-task competition (Koch, 2009). In the task-switching part, there was, however, no effect of the response sequence on task repetition trials which are used for the calculation of mixing costs.

However, we did not assess direct markers of inhibitory control in the present study. In the task-switching domain, there is converging evidence that inhibition is involved in task-switching performance (e.g., Mayr \& Keele, 2000; for a review, see, e.g., Koch et al., 2010). In dual-task research, however, inhibition has not yet been examined systematically (see Hirsch, Nolden, \& Koch, 2017, for an exception), thus remaining an important topic for future studies.

\section{WORKING MEMORY UPDATING}

The correlation analysis showed a positive relationship between mixing costs and dual-task costs, thereby indicating that these costs might rely on overlapping cognitive control processes. These costs represent performance differences between conditions in which monitoring of incoming information is necessary and conditions in which no monitoring of incoming information is required. Thus, mixing costs and dual-task costs reflect presumably cognitive control processes in terms of updating.

However, by employing univalent stimuli (i.e., stimuli linked to one task), we could not exclude the alternative explanation that subjects built a combined task set comprising stimulus-response mappings for the digit and letter tasks simultaneously (e.g., Kleinsorge \& Heuer, 1999). In this case, mixing costs would reflect higher working memory load in mixed-task blocks compared to single-task blocks, and the correlation between mixing costs and dual-task costs would rather be due to the working memory load than updating. The use of univalent stimuli in the dual-task part allowed us to study the effects of T1-T2 sequence in a traditional PRP procedure without cues. Future studies should replicate the findings of the present study based on a design that allows, despite the use of a traditional PRP procedure, the application of bivalent stimuli in the task-switching and the dual-task paradigm.

Note that mixing costs as a marker of working memory updating were also related to switch costs, switch costs in T2, and the PRP effect, which are assumed to rely on the shifting component. These correlations might indicate difficulties in isolating experimentally the shifting component in switch conditions. Successful task-switching requires the monitoring of incoming information to determine task-switches and update task sets in accordance with new tasks. Based on this line of 
argument, switch costs, switch costs in T2 and the PRP effect should, in addition to the shifting component, also rely on the updating component.

Nevertheless, the question remains why only mixing costs, and not dual-task costs, were related to performance costs that depend on the shifting component. One explanation is that, in addition to the updating component, mixing costs also measure other processes of cognitive control that are not reflected in dual-task costs, which, however, contribute to task-switching performance.

A further explanation might be related to the differences in the experimental settings. One such a difference is that in the dual-task part the two trials are separated by long ITIs, while in the task-switching part the tasks are performed in rapid succession, inducing strong between-task interference even in repetition trials. In this regard, it might be possible that, in contrast to T1-T2 repetitions, monitoring and updating occurs in repetition trials of mixed-task blocks under processing demands that are more similar to that in switch conditions. In the present study, we could only speculate on this point, meaning that more research is required.

\section{ADDITIONAL FACTORS AND THEIR RELATION TO OTHER STUDIES}

In line with the present study, Miyake and colleagues (2000) proposed that shifting contributes to switch costs. However, they found dual-task costs to be independent of all three core components, thus drawing the conclusion that, in contrast to task-switching performance, dual-task performance might rely on cognitive control mechanisms that are not related to the examined three core components. In contrast, we concluded based on our findings from an empirical approach in terms of a cross-paradigm correlation analysis between performance costs measured in highly comparable task-switching and dual-task paradigms that the core components of cognitive control are involved in dual-task performance, at least in terms of working memory updating and shifting, as indicated by the correlations between mixing costs and dual-task costs, and between switch costs and switch costs in T2 of the dual-task part.

The contradictory findings regarding the role of the core components of cognitive control for dual-task costs might be accounted for by the use of different task types. Whereas Miyake et al. (2000) used two continuous tasks (i.e., maze tracing speed test and word generation task) to be performed over several minutes and measured the number of correctly solved tasks. We employed two discrete tasks with an identical number of tasks per subject, and assessed RT and errors. Discrete tasks have a definitive observable start and end point (i.e., stimulus onset and response execution; Salvucci, 2005), and are usually separated by an ITI, allowing performance to be analyzed on a fine-grained trial-by-trial level.

In this context, Pashler and Johnston (1989) argued that, in contrast to discrete tasks, analysing performance on continuous tasks has a limited contribution to the theoretical understanding of the causes of dual-task interference because simultaneous task processing cannot be distinguished from a strategy of switching between tasks when focus- ing solely on the number of correctly solved tasks. Yet, continuous tasks have the advantage over discrete tasks that they allow for the investigation of feedback and error correction. Thus, discrete and continuous tasks can be used to study different aspects of dual-task processing, which possibly are also differently related to the core components of cognitive control.

\section{Use of Integrated Speed-Accuracy Measures}

The use of integrated measures is especially well suited when speed and accuracy are assumed to be driven by overlapping processes. Taskswitching and dual-tasking have been shown to result in performance costs in both speed and accuracy. This indicates that both these performance measures rely on the same processes (e.g., Halvorson et al., 2013; Han \& Marois, 2013; Meiran, 1996). Apart from response-selection bottleneck models that predict a PRP effect only in the RT (e.g., Pashler, 1994), dual-task models, such as the capacity sharing model (Tombu \& Jolicoeur, 2003) and the ECTVA model (Logan \& Gordon, 2001), can account for SOA effects in RTs and error rates, thus justifying the use of integrated measures. Moreover, integrated measures have already been used in the task-switching domain (e.g., Draheim, Hicks, \& Engle, 2016; Schuch \& Pütz, 2018) and the dual-task domain (Han \& Marois, 2013; Kunde, Pfister, \& Janczyk, 2012).

\section{SUMMARY AND CONCLUSIONS}

In sum, the present study represents a first step towards understanding the relationship between the cognitive control components involved in task set control by providing evidence that mixing costs and dual-task costs are markers of working memory updating, and that switch costs and switch costs in T2 are indices of the shifting component, but partly also of the updating component. Notably, we also found that the PRP effect correlated substantially $(r>.5)$ with switch costs, suggesting that the PRP effect is related to underlying shifting processes rather than being purely based on passive response queuing processes. The present study also showed that performance costs in multitasking do not measure the components of cognitive control in an isolated and differentiated manner. This is in line with the conclusion by Miyake et al. (2000), who argued that the components of cognitive control are related as well as separable. Future studies need to extend these conclusions to multitasking contexts using different types of tasks in order to establish the generality of the present conclusions. In this connection, it would also be important to focus on inhibition and to use bivalent stimuli in order to exclude working memory load as an alternative explanation for the correlation between mixing costs and dual-task costs..

\section{FOOTNOTES}

${ }^{1}$ The manipulation of specific time intervals enables the investigation of task preparation in task-switching. Preparation is analyzed by manipulating the RSI in the alternating-runs variant and by varying the cue-stimulus interval (CSI; i.e., time interval between the onset of the task cue and the presentation of the target stimulus) in the cueing 
variant. Reduced switch costs with long RSIs compared to short RSIs can be attributed to both active preparation for the upcoming task and decay of activation related to the preceding task. In the cueing variant, active preparation can be isolated by varying the RCI inversely to the CSI, while activation decay of the previous task can be isolated by varying the RCI and holding the CSI constant (see Kiesel et al., 2010, for a review). In the alternating-runs variant, subjects have to keep track of the task sequence, representing an additional working memory requirement. Here, preparation for the upcoming task can proceed immediately after response execution to the current task, and switching is driven internally. In contrast, in the cueing variant of the task-switching paradigm, switching is driven by external cues and there are no demands relating to the maintenance of the task sequence but on cue encoding. Moreover, task preparation has to wait until the presentation of the cue. Thus, these task-switching variants allow the isolation of different types of task preparation.

${ }^{2}$ Note that owing to the avoidance of immediate stimulus repetitions, the likelihood of response (side) repetitions and response (side) switches differed across task-switch trials and task-repetition trials. Task-switch trials were associated with eight stimuli, and thus, there was a 50:50 chance of the correct response. In contrast, task-repetition trials were associated only with seven stimuli because the stimulus displayed in the previous trial could not be presented again. Thus, in task-repetition trials, there was a larger likelihood for a response (side) switch than for a response (side) repetition. Implicit learning of the stimulus presentation, therefore, might have influenced switch costs by improving performance in task repetition trials.

${ }^{3}$ To explore whether faster responses with short than with long SOA were due to response grouping, we analyzed the time intervals between the response for $\mathrm{T} 1$ and that for $\mathrm{T} 2$ (i.e., interresponse interval, IRI). We defined IRIs less than $100 \mathrm{~ms}$ as indicative for response grouping (e.g., Miller \& Ulrich, 2008). Overall, there were $9.5 \%$ trials with an IRI less than $100 \mathrm{~ms}$. When excluding trials with grouped responses, the data pattern did not change, except that the trend towards an interaction of T1-T2 sequence and SOA in T1 became significant, $F(1,31)=6.05, p<.05, \eta_{\mathrm{p}}{ }^{2}=.16$. Thus, the main effect of SOA was not due to response grouping.

\section{REFERENCES}

Allport, A., Styles, E. A., \& Hsieh, S. (1994). Shifting intentional set: Exploring the dynamic control of tasks. In C. Umilta \& M. Moscovitch (Eds.), Attention and performance XV (pp. 421-452). Cambridge, MA: MIT Press.

Band, G. P. H., Jolicoeur, P., Akyürek, E. G., \& Memelink, J. (2006). Integrative views on dual-task costs. European Journal of Cognitive Psychology, 18, 481-492. doi: 10.1080/09541440500422675

Band, G. P. H., \& van Nes, F. T. (2006). Reconfiguration and the bottleneck: Does task switching affect refractory period effect? European Journal of Cognitive Psychology, 18, 481-492. doi: 10.1080/09541440500423244
Best, J. R., \& Miller, P. H. (2010). A developmental perspective on executive functions. Child Development, 81, 1641-1660. doi:

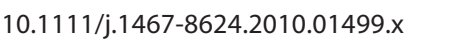

Bruyer, R., \& Brysbaert, M. (2011). Combining speed and accuracy in cognitive psychology: Is the inverse efficiency score (IES) a better dependent variable than the mean reaction time (RT) and the percentage of errors (PE)? Psychologica Belgica, 51, 5-13. doi: 10.5334/pb-51-1-5

Cohen, J. D. (2017). Cognitive control. Core constructs and current considerations. In T. Egner (Ed.), The Wiley handbook of cognitive control (pp. 3-28). Chichester, UK: John Wiley \& Sons, Ltd. doi: 10.1002/9781118920497.ch1

Declerck, M., Grainger, J., Koch, I., \& Philipp, A. M. (2017). Is language control just a form of executive control? Evidence for overlapping processes in language switching and task switching. Journal of Memory and Language, 95, 138-145. doi: 10.1016/j.jml.2017.03.005

Draheim, C., Hicks, K. L., \& Engle, R. W. (2016). Combining reaction time and accuracy: The relationship between working memory capacity and task switching as a case example. Perspectives on Psychological Science, 11, 133-155. doi: $10.1177 / 1745691615596990 \underline{\underline{W W}}$

Druey, M. D. (2014). Stimulus-category and response-repetition effects in task switching: An evaluation of four explanations. Journal of Experimental Psychology: Learning, Memory, and Cognition, 40, 125-46. doi: 10.1037/a0033868 wwW

Druey, M. D., \& Hübner, R. (2008). Response inhibition under task switching: Its strength depends on the amount of task-irrelevant response activation. Psychological Research, 72, 515-527. doi: 10.1007/s00426-007-0127-1. WWW

Duncan, J. (1995). Attention, intelligence, and the frontal lobes. In M. S. Gazzaniga (Ed.), The cognitive neurosciences (pp. 721733). Cambridge, MA: MIT Press. doi: 10.1006/cogp.1996.0008

Feng, S. F., Schwemmer, M., Gershman, S. J., \& Cohen, J. D. (2014). Multitasking versus multiplexing: Toward a normative account of limitations in the simultaneous execution of controldemanding behaviors. Cognitive, Affective, and Behavioral Neurosciences, 14, 129-146. doi: 10.3758/s13415-013-0236-9 WWW

Fischer, R., \& Dreisbach, G. (2015). Predicting high levels of multitasking reduces between-tasks interactions. Journal of Experimental Psychology: Human Perception and Performance, 41, 1482-1487. doi: 10.1037/xhp0000157 WWW

Fischer, R., \& Plessow, F. (2015). Efficient multitasking: Parallel versus serial processing of multiple tasks. Frontiers in Psychology, 6:1366. doi: 10.3389/fpsyg.2015.01366 |

Friedman, N., \& Miyake, A. (2004). The relations among inhibition and interference control functions: A latent-variable analysis. Journal of Experimental Psychology: General, 133, 101-135. doi: 10.1037/0096-3445.133.1.1 $\underline{\underline{\mathrm{WW}}}$

Grzyb, K. R., \& Hübner, R. (2013). Excessive response-repetition costs under task switching: How response inhibition amplifies 
response conflict. Journal of Experimental Psychology: Learning, Memory, and Cognition, 39, 126-139. doi: 10.1037/a0028477 WWW

Halvorson, K. M., Ebner, H., \& Hazeltine, E. (2013). Investigating perfect timesharing: The relationship between IM-compatible tasks and dual-task performance. Journal of Experimental Psychology: Human Perception and Performance, 39, 413-423. doi: 10.1037/a0029475 WWW

Han, S. W., \& Marois, R. (2013). The source of dual-task limitations: Serial or parallel processing of multiple response selection. Attention, Perception, \& Psychophysics, 75, 1395-1405. doi: 10.3758/s13414-013-0513-2 WWW

Hirsch, P., Declerck, M., \& Koch, I. (2015). Exploring the functional locus of language switching: Evidence from a PRP paradigm. Acta Psychologica, 161, 1-6. doi: 10.1016/j.actpsy.2015.07.010 WWW

Hirsch, P., Nolden, S., \& Koch, I. (2017). Higher-order cognitive control in dual tasks: Evidence from task-pair switching. Journal of Experimental Psychology: Human Perception and Performance, 43, 569-580. doi: 10.1037/xhp0000309 WWW

Hommel, B. (1988). Automatic stimulus-response translation in dual-task performance. Journal of Experimental Psychology: Human Perception and Performance, 24, 1368-1384. doi:

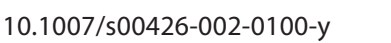

Hübner, R., \& Druey, M. D. (2006). Response execution, selection, or activation: What is sufficient for response-related repetition effects under task shifting? Psychological Research, 70, 245261. doi: 10.1 007/s00426-005-0219-8 WWW

Kiesel, A., Wendt, M., Jost, K., Steinhauser, M., Falkenstein, M., Philipp, A. M., \& Koch, I. (2010). Control and interference in task switching: A review. Psychological Bulletin, 136, 849-874. doi: 10.1037/a0019842 WWW

Kleinsorge, T., \& Heuer, H. (1999). Hierarchical switching in a multi-dimensional task space. Psychological Research, 62, 300-312. doi: $10.1007 /$ s004260050060

Koch, I. (2009). The role of crosstalk in dual-task performance: Evidence from manipulating response-code overlap. Psychological Research, 73, 417-424. doi: 10.1007/s00426-008 $-0152-8 \underline{\underline{W W}}$

Koch, I., Frings, C., \& Schuch, S. (2017). Explaining responserepetition effects in task switching: Evidence from switching cue modality suggests episodic binding and response inhibition. Psychological Research. Advance online publication. doi: 10.1007/s00426-017-0847-9. |

Koch, I., Gade, M., Schuch, S., \& Philipp, A. M. (2010). The role of inhibition in task switching: A review. Psychonomic Bulletin \& Review, 17, 1-14. doi: 10.3758/PBR.17.1.1

Koch, I., Poljac, E., Müller, H., \& Kiesel, A. (2018). Cognitive structure, flexibility, and plasticity in human multitasking-An integrative review of dual-task and task-switching research. Psychological Bulletin. Advance online publication. doi: 10.1037/bul0000144
Koch, I., Prinz, W., \& Allport, A. (2005). Involuntary retrieval in alphabet arithmetic tasks: Task-mixing and task-switching costs. Psychological Research, 69, 252-261. doi: 10.1007/s00426-0040180-y WWW

Koch, I., Schuch, S., Vu, K. P., \& Proctor, R. W. (2011). Responserepetition effects in task switching-Dissociating effects of anatomical and spatial response discriminability. Acta Psychologica, 3, 399-404. doi: 10.1016/j.actpsy.2010.07.016 WWW

Kunde, W., Pfister, R., \& Janczyk, M. (2012). The locus of tooltransformation costs. Journal of Experimental Psychology: Human Perception and Performance, 38, 703-714. doi: 10.1037/

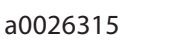

Lehto, J. E., Juujärvi, P., Kooistra, L., \& Pulkkinen, L. (2003). Dimensions of executive functioning: Evidence from children. British Journal of Developmental Psychology, 21, 59-80. doi: 10.1348/026151003321164627

Lien, M. C., \& Proctor, R. W. (2002). Stimulus-response compatibility and psychological refractory period effects: Implications for response selection. Psychonomic Bulletin \& Review, 9, 212-238. doi: 10.3758/BF03196277 $\overline{\mathrm{WWW}}$

Lien, M. C., Schweickert, R., \& Proctor, R. W. (2003). Task switching and response correspondence in the psychological refractory period paradigm. Journal of Experimental Psychology: Human Perception and Performance, 29, 692-712. doi: 10.1037/0096-

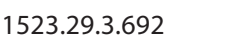

Liepelt, R., Strobach, T., Frensch, P. A., \& Schubert, T. (2011). Improved intertask coordination after extensive dual-task practice. Quarterly Journal of Experimental Psychology, 64, 1251-1272. doi: 10.1080/17470218.2010.54328 |WW|

Logan, G. D. (2003). Executive control of thought and action: In search of the wild homunculus. Current Directions in Psychological Science, 12, 45-48. doi: 10.1111/14678721.01223

Logan, G. D., \& Gordon, R. D. (2001). Executive control of visual attention in dual-task situations. Psychological Review, 108, 393-434. doi: 10.1037/0033-295X.108.2.393 WWW

Mayr, U. (2001). Age differences in the selection of mental sets: The role of inhibition, stimulus ambiguity, and response-set overlap. Psychology and Aging, 16, 96-109. doi: 10.1037/08827974.16.1.96 $\overline{\mathrm{WWW}}$

Mayr, U., \& Keele, S. W. (2000). Changing internal constraints on action: The role of backward inhibition. Journal of Experimental Psychology: General, 1, 4-26. doi: 10.10371/0096-3445.129.1.4 WWW

Meiran, N. (1996). Reconfiguration of processing mode prior to task performance. Journal of Experimental Psychology: Learning, Memory, and Cognition, 22, 1423-1442. doi: 10.1037/02787393.22.6.1423

Meyer, D. E., \& Kieras, D. E. (1997). A computational theory of executive cognitive processes and multiple-task performance: Part 1. Basic Mechanisms. Psychological Review, 104, 3-65. doi:

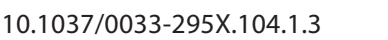


Miller, J., \& Ulrich, R . (2008). Bimanual response grouping in dualtask paradigms. Quarterly Journal of Experimental Psychology, 61, 999-1019. doi: 10.1080/17470210701434540 WWW

Miyake, A., \& Friedman, N. P. (2012). The nature and organization of individual differences in executive functions: Four general conclusions. Current Directions in Psychological Sciences, 21, 8-14. doi: $10.1177 / 0963721411429458$ [Ww]

Miyake, A., Friedman, N. P., Emerson, M. J., Witzki, A. H., Howerter, A., \& Wager, T.D. (2000). The unity and diversity of executive functions and their contributions to complex frontal lobe tasks: A latent variable analysis. Cognitive Psychology, 41, 49-100. doi: 10.1006/cogp.1999.0734 WWW

Monsell, S. (2003). Task switching. Trends in Cognitive Sciences, 7, 134-140. doi: 10.1016/S1364-6613(03)00028-7 [wWw

Oriet, C., \& Jolicoeur, P. (2003). Absence of perceptual processing during reconfiguration of task set. Journal of Experimental Psychology: Human Perception and Performance, 29, 1036-1049. doi: $10.1037 / \mathrm{a} 0022192$ www

Pashler, H. (1994). Dual-task interference in simple tasks: Data and theory. Psychological Bulletin, 116, 358-377. doi: 10.1037/00332909.116.2.220 WWW

Pashler, H. (2000). Task switching and multitask performance. In S. Monsell \& J. Driver (Eds.), Control of cognitive processes: Attention and performance XVIII (pp. 277-309). Cambridge, MA: The MIT Press. doi: 10.1002/acp.849

Pashler, H., \& Johnston, J. C. (1989). Chronometric evidence for central postponement in temporally overlapping tasks. Quarterly Journal of Experimental Psychology Section A: Human Experimental Psychology, 41A, 19-45. doi: 10.1080/14640748908402351

Rogers, R. D., \& Monsell, S. (1995). Costs of a predictable switch between simple cognitive tasks. Journal of Experimental Psychology: General, 124, 207-231. doi: 10.1037/0096-3445 .124.2.207

Rubin, O., \& Meiran, N. (2005). On the origins of the task mixing cost in the cuing task-switching paradigm. Journal of Experimental Psychology: Learning, Memory, and Cognition, 31, 1477-1491. doi: 10.1037/0278-7393.31.6.1477 WWW

Salvucci, D. D. (2005). A multitasking general executive for compound continuous tasks. Cognitive Science, 29, 457-492. doi: 10.1207/s15516709cog0000_19 www
Schubert, T. (2008). The central attentional limitation and executive control. Frontiers in Bioscience, 13, 3569-3580. doi: $10.2741 / 2950$ [WWW

Schubert, T., Fischer, R., \& Stelzel, C. (2008). Response activation in overlapping tasks and the response-selection bottleneck. Journal of Experimental Psychology: Human Perception and Performance, 34, 376-397. doi: 10.1037/0096-1523.34.2.376 WWW

Schuch, S., \& Koch, I. (2003). The role of response selection for inhibition of task sets in task shifting. Journal of Experimental Psychology: Human Perception and Performance, 29, 92-105. doi: 10.1037/0096-1523.29.1.92 WwW]

Schuch, S., \& Koch, I. (2004). The costs of changing the representation of action: Response repetition and response-response compatibility in dual tasks. Journal of Experimental Psychology: Human Perception and Performance, 30, 566-582. doi:

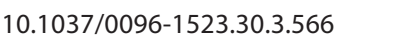

Schuch, S., \& Koch, I. (2010). Response-repetition effects in task switching with and without response execution. Acta Psychologica, 3, 302-309. doi: 10.1016/j.actpsy.2010.07.016 WWW

Schuch, S., \& Pütz, S. (2018). Mood state dissociates conflict adaptation within tasks and across tasks. Journal of Experimental Psychology: Learning, Memory, and Cognition. Advance online publication. doi: 10.1037/xIm0000530 www

Sigman, M., \& Dehaene, S. (2006). Dynamics of the central bottleneck: Dual-task and task uncertainty. PLOS Biology, 4, 12271238. doi: 10.1371 /journal.pbio.0040220 [WWW

Strobach, T., \& Schubert, T. (2017). Mechanisms of practice-related reductions of dual-task interference with simple tasks: Data and theory. Advances in Cognitive Psychology, 13, 28-41. doi: 10.5709/acp-0204-7 WWW

Tombu, M., \& Jolicoeur, P. (2003). A central capacity sharing model of dual-task performance. Journal of Experimental Psychology: Human Perception and Performance, 29, 3-18. doi: 10.1037/0096-1523.29.1.3 WwW

Vandierendonck, A. (2017). A comparison of methods to combine speed and accuracy measures of performance: A rejoinder on the binning procedure. Behavior Research Methods, 49, 653-673. doi: 10.3758/s13428-016-0721-5 wWW 


\section{APPENDIX A}

\begin{tabular}{|c|c|c|c|c|c|c|}
\hline A. Task-switching part & Ks1 $100 \mathrm{~ms}$ & & KSI effect & RSI $100 \mathrm{~ms}$ & RSI $600 \mathrm{~ms}$ & RSI effect \\
\hline & $824(37)$ & 737 (38) & 87 & $4.8(0.7)$ & $4.9(0.6)$ & -0.1 \\
\hline Repetition & $798(32)$ & $687(27)$ & 111 & $3.4(0.6)$ & $5.9(0.8)$ & -2.5 \\
\hline Single-task & $731(21)$ & $636(21)$ & 95 & $4.4(1.0)$ & $5.3(0.9)$ & -0.9 \\
\hline Switch costs & 26 & 50 & & 1.4 & -1.0 & \\
\hline Mixing costs & 67 & 51 & & -1.0 & 0.6 & \\
\hline B. Dual-task part & SOA $100 \mathrm{~ms}$ & SOA $600 \mathrm{~ms}$ & SOA effect & SOA $100 \mathrm{~ms}$ & SOA $600 \mathrm{~ms}$ & SOA effect \\
\hline $\mathrm{T} 1-\mathrm{T} 2$ switch for $\mathrm{T} 1$ & $887(57)$ & $880(58)$ & 7 & $4.7(0.6)$ & $7.1(0.8)$ & -2.4 \\
\hline $\mathrm{T} 1-\mathrm{T} 2$ repetition for $\mathrm{T} 1$ & $872(56)$ & $891(63)$ & -19 & $4.6(0.7)$ & $7.9(1.0)$ & -3.3 \\
\hline Switch costs for $\mathrm{T} 1$ & 15 & -11 & & 0.1 & -0.8 & \\
\hline T1-T2 switch for T2 & $1470(98)$ & $648(44)$ & 822 & $7.7(1.1)$ & $9.3(1.0)$ & -1.6 \\
\hline $\mathrm{T} 1-\mathrm{T} 2$ repetition for $\mathrm{T} 2$ & $1395(87)$ & $588(35)$ & 807 & $7.4(1.1)$ & $8.8(1.2)$ & -1.4 \\
\hline Single-task & $552(10)$ & $521(10)$ & 31 & $3.2(0.5)$ & $4.6(0.6)$ & -1.4 \\
\hline Switch costs for T2 & 75 & 60 & & 0.3 & .06 & \\
\hline Dual-task costs & 843 & 67 & & 4.1 & 4.2 & \\
\hline
\end{tabular}

Note. In the task-switching part, there were speed-accuracy trade-offs with regard to the main effect of RSI (mixing-cost contrast: RSI effect of 103 ms in the RT data, $F[1,31]=116.24, p<.001, \eta_{\mathrm{p}}{ }^{2}=.79$, and reversed RSI effect of $-1.7 \%$ in the error data, $F[1,31]=21.93, p<.001, \eta_{\mathrm{p}}{ }^{2}=.41$; switch-cost contrast: RSI effect of 99 ms in the RT data, $F[1,31]=66.43, p<.001, \eta_{\mathrm{p}}{ }^{2}=.68$, and reversed RSI effect of $-1.3 \%$ in the error data, $F[1,31]=8.06, p<.01, \eta_{\mathrm{p}}{ }^{2}=.21$, ) and the interaction of RSI and trial type (switch-cost contrast: switch costs of $26 \mathrm{~ms}$ with short RSI and switch costs of $50 \mathrm{~ms}$ with long RSI in the RT data, $F[1,31]=4.30, p<.05, \eta_{\mathrm{p}}{ }^{2}=.12$, as well as switch costs of $1.4 \%$ with short RSI and switch costs of $-1.0 \%$ with long RSI in the error data, $\left.F[1,31]=10.31, p<.01, \eta_{\mathrm{p}}{ }^{2}=.25\right)$. In the dual-task part, there was a speed-accuracy trade-off in T2 with respect to the main effect of SOA (PRP effect of $815 \mathrm{~ms}$ in the RT data, $F[1,31]=152.96, p<.001, \eta_{\mathrm{p}}{ }^{2}=.83$, and a reversed PRP effect of $-1.5 \%$ in the error data, $\left.F[1,31]=5.87, p<.05, \eta_{\mathrm{p}}{ }^{2}=.16\right)$. 